\title{
Lateral Stirring of large-scale tracer fields by altimetry
}

Guillaume Dencausse, Rosemary Morrow, Marine Rogé, Sara Fleury

LEGOS, 14 avenue Edouard Belin, 31400 Toulouse, France

June 2013

Ocean Dynamics

\begin{abstract}
Ocean surface fronts and filaments have a strong impact on the global ocean circulation and biogeochemistry. Surface Lagrangian advection with time-evolving altimetric geostrophic velocities can be used to simulate the submesoscale front and filament structures in large-scale tracer fields. We study this technique in the Southern Ocean region south of Tasmania, a domain marked by strong meso- to submesoscale features such as the fronts of the Antarctic Circumpolar Current (ACC). Starting with large scale surface tracer fields that we stir with altimetric velocities, we determine 'advected' fields which compare well with high resolution in-situ or satellite tracer data. We find that fine scales are best represented in a statistical sense after an optimal advection time of $\sim 2$ weeks, with enhanced signatures of the ACC fronts and better spectral energy. The technique works best in moderate to high EKE regions where lateral advection dominates. This technique may be used to infer the distribution of unresolved small scales in any physical or biogeochemical surface tracer that is dominated by lateral advection. Submesoscale dynamics also impact the subsurface of the ocean, and the Lagrangian advection at depth shows promising results. Finally, we show that climatological tracer fields computed from the advected large scale fields display improved fine scale mean features, such as the ACC fronts, which can be useful in the context of ocean modelling.
\end{abstract}




\section{Introduction}

The role of mesoscale and submesoscale dynamics in setting frontal structures in the upper layers of the ocean has recently drawn increasing focus. Satellite data have revealed the presence of ocean surface filaments associated with strong fronts - or sharp gradients - in various sea surface properties, including SST (Turiel et al.,2005; Hughes et Ash, 2001), dynamic height gradients (Sokolov and Rintoul; 2007, Dencausse et al., 2010) or chlorophyll content (Lehahn et al., 2007). Horizontal dynamics were shown to contribute to horizontal mixing, with impacts on local biology (Abraham, 1998; Abraham et al., 2000). Moreover, the intense horizontal gradients across submesoscale fronts are also associated with strong vertical circulation cells. The ensuing vertical displacements impact on the local biology through import and export from the photic layer (Lévy et al., $2001 \& 2005$ ), and also modify water mass properties down to - and sometimes beyond - the mixed layer depth, impacting on water mass formation (Paci et al., 2005; Sallée et al., 2006).

These studies reveal the significant role of submesoscale activity around oceanic fronts and its impact on local and global ocean circulation and biology. They also stress the need for a better understanding of fronts and submesoscale physics. While ocean circulation models are crucial for predicting the evolution of the earth's climate, model parameterizations for dynamics at submesoscales need to be further developed (Fox-Kemper et al., 2008, Ferrari et al, 2008; Thomas and Ferrari, 2008; Le Sommer et al., 2011). A prerequisite for such improvement is to further observe and understand the impacts of mesoscale and submesoscale dynamics at regional to larger scales. Today, satellite and in-situ data are providing only partial coverage of these processes. In-situ underway observations from ship cruises, particularly from thermosalinographs (TSG), offer sufficient horizontal resolution along each transect for studying fronts locally, at scales of a few km (Chaigneau and Morrow, 2002; Desprès et al., 2011). Argo floats - which have been deployed in increasing number since 2005 - provide a more global vision of the larger scale processes $(>300 \mathrm{~km}$ ) but do not resolve smaller mesoscale eddies or fronts. Neither data set offers global homogeneous spatial and temporal coverage of mesoscale or submesoscale dynamics, limiting the scope of analyses from in-situ data alone. Satellite observations of sea surface color, or temperature can resolve fronts at submesoscales with sufficient horizontal resolution, the only setback being occasional cloud cover. Microwave satellite SST provides global coverage, but only of mesoscale resolution. Time series of SST maps based on combined microwave and infrared 
data are becoming available, and offer interesting perspectives in this domain of study.

There is however no time series of sea surface salinity (SSS) or other tracers such as carbon or nutrients, with equivalent spatial resolution. It is essential to have fine-resolution SSS time series, along with SST fields, since both parameters control upper ocean buoyancy, which is a key parameter for better understanding the upper ocean stability and mixed layer structure, and the 3D upper ocean circulation at submesoscales (Klein and Lapeyre, 2009). The SMOS mission, launched in 2009, and the Aquarius mission, launched in June 2011, are starting to provide global observations of SSS. However, the horizontal resolution of the satellite SSS products is not expected to resolve the mesoscale or submesoscale processes.

Altimetry can provide a key dynamical basis for studying mesoscale to submesoscale processes. Even though the mapped altimetric geostrophic currents only resolve the larger Eulerian mesoscale field, the temporal evolution of these 2D fields has been shown to reveal smaller filamentation (d'Ovidio et al, 2009), generated by the time-evolving dynamics. Recent work by Despres et al (2011) has shown that mesoscale and some submesoscale SSS patterns in the North Atlantic Ocean could be quite successfully inferred from large scale surface 2D tracer fields using a surface Lagrangian advection technique. This technique is based on the lateral stirring of a tracer field using the Lagrangian advection of surface particles calculated from the time-evolving altimetric velocity fields. This lateral stirring creates filaments and fronts at much smaller scales, i.e., the advection sharpens the frontal gradients, whilst other processes (diffusion, mixing) tend to smooth them out. The longer the advection time, the more fine scales are introduced in the tracer field, so the choice of the advection time is crucial. This technique of applying lateral stirring to a tracer field is quite simple, and works well in regimes where the lateral advection dominates. There are potential applications for simulating smaller scale patterns in large scale tracer products, including SMOS or Aquarius satellite SSS fields, but also large-scale gridded carbon or nutrient fields.

In this study, we apply the technique of Despres et al. (2011) to a Southern Ocean domain south of Tasmania. This region has strong deep reaching fronts which are associated with the maximum transport of the main jets of the Antarctic Circumpolar Current (e.g. Sokolov and Rintoul, 2010). Their mean paths are represented in Figure 1. These fronts separate distinct polar water masses (Orsi et al., 1995) and regions of strong air-sea fluxes. The fronts have been monitored in a circumpolar sense using SST data (Hughes and Ash, 2001) or altimetric proxies (Sallee et al., 2008; Dencausse et al., 2010). However, the 
signature of these fronts in terms of SSS is less well known, due to the poor space-time coverage of SSS data. The region south of Tasmania was chosen to evaluate the lateral stirring technique since repeated underway sampling of SSS and SST data has been routinely obtained over nearly 20 years from the SURVOSTRAL project (see Figure 1). This project collects up to 10 underway thermosalinograph transects per year between Hobart, Australia and the French Antarctic base at Dumont D’Urville, starting in 1993. The 1-minute underway SSS and SST data allows us to accurately measure the position and intensity of the surface fronts (Chaigneau and Morrow, 2002). This data set will be used to help us evaluate the satellite-based fine-resolution tracer products.

The main objective of our study is to reconstruct finer-scale surface SSS fields, to complement the high-resolution satellite SST fields that are already available. We will evaluate whether these reconstructions provide better frontal positioning and cross-frontal gradients on a weekly or seasonal basis, compared to the lower resolution tracer fields. We start with initial large scale tracer fields from a gridded weekly temperature and salinity product, calculated using an objective analysis of in-situ data - mainly Argo floats and hydrographic measurements from Coriolis (http://www.coriolis.eu.org). The fields are then stirred horizontally by altimetric geostrophic currents to produce fine-resolution $2 \mathrm{D}$ tracer fields. These advected fields are then compared to high resolution underway thermosalinograph data from the repeat SURVOSTRAL line (Chaigneau and Morrow, 2002), as well as 2D high resolution AVHRR infrared radiometer SST maps. Since the large horizontal scales of the gridded Coriolis data and upcoming SMOS or Aquarius SSS fields will be similar, the aim is to evaluate the reconstruction of meso- to submesoscales that could be applied for future satellite salinity products.

Finally, while we have some direct satellite observations of mesoscale and submesoscale dynamics at the ocean surface, the same is not true of subsurface dynamics below the mixed layer. Some techniques are being developed to project the high resolution surface density or temperature fields vertically into the ocean interior (Klein and Lapeyre, 2009, Guinehut et al, 2004). For data-based products, only larger scale subsurface temperature and salinity fields are available from in-situ observations, such as the Coriolis objective analysis fields mentioned above. Better knowledge of finer scales patterns within and below the mixed layer would be of scientific interest, for a better understanding of mixed layer dynamics and water mass formation and for model initialisation or validation. Hence the 
Lagrangian advection technique used in this study will be tested on subsurface large scale tracer fields from Coriolis, and the results compared to in-situ data.

\section{Data and methods}

\section{Lagrangian advection technique}

Low resolution SST and SSS tracer fields at the start time of an advection are interpolated onto a fine $0.04^{\circ}$ particle grid. We then perform a Lagrangian advection, and each particle is advected with its velocity and position computed every 3 hours (see illustration Figure 2). The Lagrangian trajectories of the particles are then computed and each particle trajectory is assigned an initial tracer value, which it then carries along its pathway to its final position (see Figure 2). The advected tracer field is hence meant to simulate the tracer field on the final day of advection, as demonstrated by d'Ovidio et al (2009) and Despres et al (2011). As mentioned earlier, this advection is "passive", in that no tracer modifications are introduced during the advection period - such as those resulting from frontogenesis, air-sea exchanges, or horizontal and vertical mixing. The method simply applies realistic lateral stirring of the initial tracer field. The reconstruction of fine scales, down to $\sim 10 \mathrm{~km}$ submesoscale filaments, will depend on the accuracy and smoothing of the initial tracer fields, and on the accuracy of the horizontal advection fields. Furthermore, the technique leaves out other physical factors affecting frontal distribution (e.g. Ekman transport, mixing), and we may lead to incorrect positioning of the individual fronts generated with the technique. This will be examined in detail in this paper.

As flow convergence at a front strengthens the tracer gradients, an ageostrophic circulation appears - which is ignored with this technique - that accelerates the frontal generation (Hoskins and Bretherton, 1972).

\section{Horizontal velocity data used for the advection}

The velocity fields used for the horizontal Lagrangian advection are from altimetric data, over the period 2002-2007. They were produced by Ssalto/Duacs and distributed by Aviso, with support from CNES (http://www.aviso.oceanobs.com/duacs/). They consist of weekly global 1/3 degree gridded fields of surface geostrophic velocities calculated from sea surface height (SSH) fields also distributed by Aviso. The SSH is an absolute dynamic topography, which consists of a SSH anomaly field and of a mean dynamic topography field from Rio et al (2009). These eulerian maps can resolve the larger mesoscale eddy field $>150$ 
km (Le Traon et al, 2001; Dussurget et al, 2011, Dibarboure et al., 2011), but their Lagrangian evolution can provide information on the submesoscales. For our study, these weekly Aviso altimetric currents were interpolated linearly onto a finer space-time grid, at 3-hour intervals, and onto a $0.04^{\circ}$ grid.

\section{Tracer data for the Initial Fields}

The large scale SSS and SST tracer fields used as initial conditions are from an objective analysis of temperature and salinity data from various in-situ measurements around the world, including Argo float profiles. Global Ocean Surface Underway Data (GOSUD) data are included from 2009 onward. Weekly products are mapped onto 3-D grids with 1/4 degree horizontal spacing and between 59 and 152 vertical levels depending on the period. We will call this data 'Coriolis SSS and SST data' and study the period 2002 to 2007. They are available from the Coriolis website (http://www.coriolis.eu.org), where they are referred to as Global Ocean - Real Time In-situ Observations Objective Analysis. These data were collected and made freely available by the Coriolis project and programmes that contribute to it.

Analysis data

To analyse the advected fields, we compare them with the high resolution underway SSS and SST data from the SURVOSTRAL repeat cruise south of Tasmania (http://www.legos.obs-mip.fr/soa/salinite/SURVOSTRAL/) aboard the Astrolabe (IPEV). Underway SSS and SST data are measured by a Seabird Thermosalinograph (TSG) and are available every minute; the data processing is described by Chaigneau and Morrow (2002). The vessel links Hobart and the French Antarctic base in Dumont d'Urville (see the cruise track on Figure 1), although measurements can only be made as far south as the seasonal ice cover permits. There are usually five round trips per year - and thus twice as many transects taking place between October and March. High resolution repeat XBT measurements from the same cruise campaign are also used in this study to evaluate the subsurface advected fields (http://www.legos.obs-mip.fr/fr/projets/SURVOSTRAL/).

Composite satellite SST data from the Advanced Very High Resolution Radiometers (AVHRR) are used as analysis data for the advected microwave SST fields. As cloud cover can hinder ocean surface coverage, we will be using 6-day composites, as they offer sufficient 
coverage for our purposes while capturing the smallest scales investigated in the study. We use a regional AVHRR product, developed by the CSIRO in Australia (http://imos.aodn.org.au/oceancurrent/ten_years_of_SST/). The horizontal grid spacing is $0.036^{\circ}$ in latitude and $0.042^{\circ}$ in longitude.

Finally, we use microwave satellite SST obtained from an optimal interpolation of AMSR-E microwave satellite radiometer data (http://www.ghcc.msfc.nasa.gov/AMSR/) for further validation. Daily fields are available from June 2002 to present, and are mapped on a $25 \mathrm{~km}$ grid although the effective resolution of mesoscale structures will be larger $(>100 \mathrm{~km})$.

\section{Advection of large scale sea surface tracer fields}

We will test the Lagrangian advection technique south of Tasmania, focusing on the 5year period 2002-2007 when both the large scale Coriolis SST and SSS fields, and thermosalinograph data from the SURVOSTRAL mission are available.

An important issue is the choice of an appropriate advection time. The primary goal is here to obtain tracer fields with structures down to the submesoscales that compare well with in-situ observations, at least in a statistical sense. If the advection time is too short, the lateral stirring will be insufficient to generate such scales. If this time is too long, the missing terms which can modify the SST or SSS will become important (e.g. air-sea fluxes, vertical and horizontal mixing, frontogenesis dynamics), and the submesoscale might be over-represented, while the larger scales may also drift away from the observations. The advection time which gives the best results will depend on 1) the accuracy and smoothing of the initial tracer field, 2) the accuracy and smoothing of the altimetric velocities, and 3) the balance of physical processes which contribute to the evolution of the tracer field (i.e., mean and eddy horizontal advection, vertical advection, diffusion and mixing, air-sea fluxes). In addition, each surface tracer, SSS or SST, may have a different response time. For example SST, being coupled with the air-sea fluxes, responds quickly to air-sea fluxes and also tends to dampen air-sea flux anomalies. However, given that our initial tracer fields are only distributed weekly and that the SSS and SST are strongly correlated at the polar fronts (Sokolov and Rintoul, 2006; Morrow et al., 2008), we will analyse the SST and SSS response times together.

The importance of adequately choosing the advection time appears clearly when we consider the 2D SST fields obtained after various advection times, and compare them with high resolution 6-day composite AVHRR SST images. An example in Figure 3 shows the 
Coriolis and AVHRR SST fields on 20 February 2007 (panels a \& d), compared to advected fields which have had 6 or 13 days of advection leading up to the 20 February 2007 (panels b $\&$ c). We can see the increasing development of mesoscale and finer scale structures such as filaments with increasing advection time. Mesoscale features captured in the AVHRR image (d) are clearly absent in the Coriolis field (a). The 6-day advection (b) simulating the SST on 20 February 2007 already reproduces some of the mesoscale patterns present in the AVHRR image. (Note that the SST advected values come from the 14 February 2007, 6 days before the final advection time). One of the most visible patterns in this case is the patch of $8^{\circ}-10^{\circ} \mathrm{C}$ water protruding into colder waters at approximately $51^{\circ}-53^{\circ} \mathrm{S}$ and $142^{\circ}-146^{\circ} \mathrm{E}$. The longer 13-day advection (c) resembles the 6-day advection pattern, but displays more small scale structures, with long and narrow filaments with sharper SST gradients. This gradual development of smaller scales using this technique has been previously observed and can also be revealed using Lyapunov exponents (Lehahn et al., 2007, d'Ovidio et al., 2009).

To further analyse the advected large scale Coriolis tracer fields and to determine an optimal advection time, we compare the 2D advected fields of SST and SSS with the underway in-situ data. We calculate a set of advection periods leading up to the median date of each cruise. The transect from Hobart to Antarctica takes $\sim 6$ days - we are mainly interested in the Subtropical and Subantarctic Fronts which lie between $47-54^{\circ} \mathrm{S}$ (see Figure 1), so the median date is chosen to represent the in-situ section centred on this latitude domain. We start the advections from earlier weekly Coriolis fields, and perform the advection up until the transect's median date. The $2 \mathrm{D}$ advected fields are then projected onto the TSG measurement points along the cruise line.

We have compared the advected fields with all of the in-situ transects over the 20022007 period. We have chosen two dates to illustrate different features in Figure 4, where SSS and SST from TSG and Lagrangian advection are plotted for transects centred on 9 November 2006 (Figure 4, left panels) and 1 January 2005 (Figure 4, right panels).

The plots for 9 November 2006 illustrate a case where the advection technique provides good results, at the beginning of the summer heating cycle. The black curves show the SST and SSS measured with the TSG, and show two main fronts. The sharp gradients separating different hydrological properties, centred on $\sim 46.5^{\circ} \mathrm{S}$ and $\sim 51.5^{\circ} \mathrm{S}$, correspond to the Subtropical Front (STF) and Subantarctic Front (SAF) respectively. Superimposed are smaller scale perturbations with sharp gradients, which can be associated with submesoscale 
fronts and filaments. The blue curves in the plots correspond to the projection of the advected tracer fields onto the measurement positions. The top panel corresponds to a 1-day advection, which is very close to the SST and SSS fields from the original Coriolis products. Clearly the STF and SAF are not resolved in those two fields, except for a very broad, smoothed SAF that appears in the SST and SSS fields.

These plots also show the growing importance of submesoscale fronts with advection time. While none are present in the case of a 1-day advection (top panel) - and hence in the Coriolis fields - their position and amplitude compares well with those in the TSG data in the case of a 15-day advection (middle panel). However, the number and amplitudes of these small-scale fronts are clearly too high after a 36-day advection (bottom panel). The rms differences between the in-situ measurements and advected fields, calculated for all points along this 9 November 2006 transect, also show a minimum for the 15 day advection time in both SST and SSS (see values in each plot of Figure 4, left panel). This preliminary comparison hints at an optimal advection time of $\sim 2$ weeks for a realistic statistical reconstruction of scales down to submesoscales. We note that the increased development of submesoscale fronts with advection time is also apparent for the formation of filaments in the 2D plots in Figure 3. The submesoscale fronts observed along the cruise line are a signature of the crossing of such filaments.

The amplitudes and positioning of the ACC fronts in the advected fields (Figure 4), do not always coincide with those measured with the thermosalinograph, even in the most realistic case of a $\sim 2$ week advection. For example, the advected position of the Subantarctic Front is well positioned in SST but is slightly too far south in SSS. This offset in the observed SSS and SST frontal positions has been observed from the in-situ data (Chaigneau and Morrow, 2002) where the surface SSS front was slightly north of the subsurface temperature fronts, which was attributed to the Ekman northward advection of the SSS front. The altimetric lateral stirring includes only the geostrophic component of the horizontal advection (no Ekman contribution), and will be driven by deeper reaching dynamics that have a stronger impact on the surface height gradients. So this offset may have a dynamical cause.

The Lagrangian advection technique can sometimes introduce biases, as illustrated in Figure 4 (right panel) for 1 January 2005, in the middle of the summer heating cycle. In this case, the rms differences grow with advection time in the case of SST. The rms value is minimal for SSS for a 38-day advection, but an excessive number of frontal features have 
developed, so that the primary goal of a good statistical frontal reconstruction is not satisfied with this advection time. If we aim to use this technique to produce quality SSS maps, the biases introduced by the advection would need to be reduced. The biases that appear between the TSG data and the advected tracer fields can be classified in two categories. The first, which we name "advection bias", comes from the advection technique itself. The second has to do with the quality of the initial tracer fields, and is referred to as "tracer bias".

To explain the tracer bias, consider the top plots of Figure $4 \mathrm{~b}$, where the blue curves represent the advected tracer field projections along the cruise line after only a 3-day advection. Little stirring has occurred at that point, so that the curves resemble the original Coriolis fields. South of $54^{\circ} \mathrm{S}$, the Coriolis fields already show offsets of about $1^{\circ} \mathrm{C}$ and 0.1 psu. These biases are important considering the $<4^{\circ} \mathrm{C}$ and $<0.3 \mathrm{psu}$ annual variations in this domain in the Coriolis fields. North of $47^{\circ} \mathrm{S}$, the SSS bias also reaches $0.4 \mathrm{psu}$, which is more than the annual variability in the Coriolis SSS fields. This tracer bias comes from the lack of in-situ data used to construct the mapped tracer fields. Very few Argo floats sample the region south of $54^{\circ} \mathrm{S}$ close to the seasonal ice edge at $60^{\circ} \mathrm{S}$, explaining the high bias there. More floats are present northward. However, the SSS bias north of $47^{\circ} \mathrm{S}$ is explained by the narrow tongue of saline and warm water which flows along the south and east Tasman coasts - a signature of the East Australian Current Extension, or 'Tasman Outflow' (Cresswell, 2000) which is apparent in the AVHRR image for 20 February 2007 in Figure 3. This tongue of water is too narrow to be well resolved by the large-scale Argo data and so is missing from the fields obtained with an objective analysis of in-situ data.

Other biases, which are evident on 1 January 2005 (Figure 4, right panel), result from the advection technique. As explained earlier, different dynamical factors affecting SSS or SST evolution are absent in our method. In the example on the right panel of Figure 4, the increase in rms differences with increasing advection time results from a clear large scale drift of the advected fields from the thermosalinograph data as the advection time increases (top to bottom panels). Amongst the different thermodynamical and dynamical processes not taken into account during the advection, two could contribute the most to such a drift. One is the lack of Ekman currents in the velocity advection fields, which could contribute to the transport at various scales across the strong meridional tracer gradients. The Ekman transport brings cool, fresh waters northward, and may explain some of the latitudinal offsets in the SSS frontal positions. The second and main factor is the absence of air-sea exchanges. We 
keep the initial tracer values fixed during the particle advection, and this example in midsummer is during the strong heating cycle. At all latitudes, the advected SST becomes progressively colder than the equivalent in-situ values for January. This is because the initial conditions are set at their mid-December values for the 17-day advection, or mid-November values for the 38-day advection. There is a big change in surface temperature during this period from the summer heating, which is not accounted for by our advection method. Similarly, the cumulated summer precipitation and sea-ice melt is missing from the SSS advection south of the SAF. In addition, frontogenesis mechanisms leading to strong vertical transports and mixing at the fronts are not taken into account and may be a source for error in the vicinity of fronts.

\section{Time evolution of the tracer and advection biases}

The results presented in Figures 3 and 4 are promising. However, if we aim to use this technique to produce time series of finer scales SSS maps, we need to analyse these biases in more detail. Figure 5 shows the bias between the underway in-situ data and advected SSS and SST fields over the entire 2002-2007 period. For each cruise transect, we define this bias as the difference - averaged over a number of measurement points - between the thermosalinograph data and the value of the advected tracer field interpolated onto the data point. This bias is the sum of the tracer bias and the advection bias. However, as noted above, the tracer bias, which varies with the quality of the original large scale tracer field, depends on the oceanic region. Hence we define three subdomains in which we calculate the bias (as shown in Figure 5). The first domain in the Subtropical Zone (STZ) extends from Tasmania $\left(44^{\circ} \mathrm{S}\right)$ to the average position of the STF $\left(47^{\circ} \mathrm{S}\right)$. The STF coincides with the southernmost penetration of the EAC Extension, where tracer bias can be strong as mentioned previously. The second domain is the Subantarctic Zone (SAZ), between the average latitudes of the Subtropical and Subantarctic Fronts - from $47^{\circ} \mathrm{S}$ to $50^{\circ} \mathrm{S}$ - where Argo floats are numerous and the initial tracer bias appears to be less important. The third domain is in the Antarctic Zone (AZ) from $55^{\circ} \mathrm{S}$ to $58^{\circ} \mathrm{S}$, south of the average position of the Polar Front, where the tracer bias in the Coriolis fields can also be important due to the lack of Argo float data.

A quantitative analysis of the 2D SST biases relative to AVHRR 2D products is presented in the Appendix. Based on the minimization of the total bias, we find an optimal advection time of $\sim 2$ weeks in the region of study, which appears well suited for the 
production of 2D SSS maps in our region. This optimal advection time also corresponds to the integration time that best reproduces the main fronts, as observed earlier from Figure 4.

Here we use a 2 week advection time to study the time evolution of the bias relative to the TSG measurements. Note that the Coriolis fields are distributed at a weekly rate, yet the in-situ SST/SSS data occur on one field date, which can occur within $14 \pm 3$ days of the initial tracer fields. The time evolution of the total bias (tracer + advection) is calculated within each subdomain and for advection times of $14 \pm 3$ days, as shown in Figure 5 (in colour). Also plotted is an estimation of the mean tracer bias for each mission, which is calculated as the mean difference between the large scale Coriolis fields for all transect dates and the TSG data, at the measurement positions (Figure 5, in black). Since the Coriolis fields are only available weekly, the two nearest fields are linearly interpolated in time onto the transect date.

The time evolution of SST and SSS total and tracer biases show a general decrease over the 2002-2007 time period studied, which depends on the region and tracer. The decrease in tracer bias indicates an improvement of Coriolis tracer fields in the region of study, explained by the gradual increase in the number of Argo floats deployed, as they are the main weekly contribution to the in-situ large-scale tracer fields. One significant exception to this tendency is in the STZ (top panels), where a decrease in biases - particularly for SSS - up until 2007, is followed by large biases during the SURVOSTRAL campaign in austral summer of 2007-2008. However, examining SST images from AVHRR or AMSRE, we find a distinctly warmer Tasman outflow signature south of Tasmania in the summer of that year than in previous years (not shown). The objective analysis used to produce the Coriolis fields smoothes out some of the sharpest tracer gradients, probably explaining the large differences with in-situ measurements for that anomalous year.

The part of the bias that is due to the advection technique can also be identified in the time evolution plots of the bias. This part of the bias corresponds to the difference between the total biases (colored dots in each plot) and the mean tracer bias (black line). Biases in the three subdomains, and for both SSS and SST, display seasonal cycles - at least over the time of year when the campaign is held, from late October to early in March on average ( 4-5 months). These cycles are clearest in the AZ, with local bias extrema in mid-December for both SSS and SST. Bias variations over the $~ 4-5$ month cycles have magnitudes comparable to the overall bias drift over the 2002-2008 period - for SSS and SST - making this part of the bias particularly significant. The origin of these seasonal cycles in the bias can be explained 
when examining the time rate of change of the Coriolis tracer fields, shown for example for the Antarctic Zone (Figure 5, bottom panel). The plot shows sharp peaks in temporal gradients at the end of December. As the advected fields are obtained by passively stirring the Coriolis fields from $14 \pm 3$ days earlier, the strong heating and freshening that has intervened during the advection time over early summer has not been accounted for. There is a clear correlation between the seasonal cycle in the bias and the strength of temporal gradients in SST and SSS.

The three subdomains show differences in the time evolutions of biases.For all regions, the SST biases are negative, indicating that the advected fields remain too cool and are not adapting to the summer heating at all latitudes. The salinity fields are more complex. The slight negative bias in the STZ means the advected fields are too fresh, and are missing some of the salty Tasman Sea water in the north - ie this is due to the tracer bias. The positive salinity bias in the AZ means that the advected fields are too salty, and here they are missing some of the freshwater coming from the melting sea-ice in late spring, again due to the tracer bias. This is associated with the negative peak in the temporal SSS gradient during the same period. The main improvement in the advection technique is for the SSS fields in the SAZ, where the fronts and the geostrophic convergences are strongest.

One point is clear with this analysis: the tracer bias component improves over time as knowledge of the background large-scale field improves, so this bias has the potential to be reduced over time (as the Argo data coverage improves in the Coriolis products). However the advection bias component is method-based and does not decrease over the period of study. This can be inferred from the difference between the mean total and tracer bias for each campaign (black and colored lines in Figure 5), which remains fairly constant over time. There is also no decrease in the time evolution of the rms values of the difference between advected fields and TSG data (not shownIt may be possible to limit the advection bias, by introducing corrections which account for the missing physics, such as a diffusion term, or evolving air-sea fluxes during the Lagrangian advection. This will be discussed further in section 7 .

\section{Reconstruction of mesoscales to submesoscales}

The most stringent test of the advection technique was to compare the individual advected maps with in-situ transects at one date, where a small ofsset in position can lead to 
large point-to-point errors. Here we will consider how the technique may improve the 2D fields in statistical averages. Here, we calculate spectra in wavenumber space from the 2D advected SST fields, and compare the results with independent satellite SST fields, from both microwave AMSR-E sensors and infrarouge AVHRR sensors. This will give us information on the statistical representation of mesoscales to submesoscales, not on the exact geographical correspondence of fronts and filaments.

The first power spectra are calculated with the large scale SST Coriolis fields that are used for advection. We then compute the spectra for the advected fields, for each of the 7, 14, 21 and 28 day advection times. Finally, we calculate the spectra for SST from AMSR-E satellite data, and from AVHRR satellite data. The AVHRR data will serve as a reference for small scales, although the noise impatcs at scales of $\sim 10 \mathrm{~km}$ or less. The spectra are calculated over a $10^{\circ}$ by $10^{\circ}$ domain $\left(141^{\circ} \mathrm{E}\right.$ to $151^{\circ} \mathrm{E}$ and $46^{\circ} \mathrm{S}$ to $\left.56^{\circ} \mathrm{S}\right)$, and for the year 2005 . Hence the spectra obtained correspond to both spatial and temporal means for this period and domain.

The mean meridional power spectra obtained (not shown) are more energetic than the zonal spectra, owing to the dominant meridional SST gradient within the ACC. However, the meridional spectra are all very similar and do not vary much with advection time. On the other hand, significant differences in energy levels appear in the detrended zonal spectra, as shown in Figure 6. The AVHRR data are more energetic than the Coriolis products at all scales spanned in the spectra, i.e. $\sim 10 \mathrm{~km}$ to $\sim 1000 \mathrm{~km}$, although the difference is most significant at the smaller scales, as expected. The microwave AMSR-E fields have equivalent zonal energy to the AVHRR fields at scales larger than $300 \mathrm{~km}$, but their energy levels drops closer to the smoothed Coriolis fields for scales smaller than $100 \mathrm{~km}$, while still being more energetic (Figure 6a).

The zonal spectra for the advected Coriolis SST fields show energy levels that increase with advection time (Figure 6b). The energy gain for the 7 day advection, compared with the non-advected Coriolis fields, is strongest at large scales ( 100 km to $\sim 1000 \mathrm{~km}$ range), this advection time being too short to generate smaller scale fronts. The important energy gain at large scales results from the large scale meridional advection of the tracer fields. Then, comparing the spectra successively by increasing advection time, we see that the energy increases progressively towards the smaller scales.

These mean zonal spectra also point to $\sim 14$ days as an optimal advection time. Energy 
levels for the 14 day advection best compare with those of AVHHR at scales down to 100 $\mathrm{km}$. These 14-day advected fields also have energy levels between the mesoscale AMSR-E SST and the finer-scale AVHRR SST, for scales from 10-100 km wavelength. Even though longer advection times appear to increase the temperature spectral energy levels at smaller scales, they also lead to excessive spectral energy levels at larger scales, which are not dissipated by our passive advection scheme.

In summary, this section suggests that when using this technique with an advection time of $\sim 14$ days, mesoscales down to the $\sim 100 \mathrm{~km}$ level are correctly reproduced in a statistical sense, and scales down to $10 \mathrm{~km}$ are improved. Comparisons with in-situ TSG data show that the main ACC fronts are generally well reproduced in cross-frontal gradients and positioning, while secondary filaments will only be reproduced statistically, and not their exact positions or associated gradients. Also, the missing physics and errors in the tracer and geostrophic velocity fields at the northern and southern boundaries can sometimes introduce significant errors, hindering the use of this technique for the production of individual SSS maps. The errors will be particularly strong in periods of strong air-sea exchanges - which are not accounted for in this passive approach, as well as in regions of low EKE where lateral stirring is not dominant in the evolution of surface SSS.

\section{Improvement of tracer climatologies}

The Lagrangian advection technique can also be used to improve our knowledge of the tracer's mean states, which are frequently used to constrain or validate ocean circulation models, or to set their initial conditions. Mean fronts across the ACC have sharp gradients that are geographically positioned by the strong bathymetric constraints on the flow (Hughes and Ash, 2001; Sokolov and Rintoul, 2007). Climatological tracer fields constructed from sparse in-situ profiles will smear these sharp gradients spatially. Since the Lagrangian advection introduces finer scales in a tracer time series through geostrophic convergence, the mean tracer pattern computed from the advected fields can also display finer scale patterns. In addition, some of the problems with the advection bias may be reduced with longer term averaging. Hence, Lagrangian advection could be used to improve the resolution of fronts in the mean surface tracer patterns.

Figure 7, top left panel, shows the time-averaged SST fields for the year 2005. The mean SST from the weekly Coriolis fields displays large scale features (Figure 7a). The sharp 
gradients associated with the ACC fronts are absent, as they were scarcely resolved in the weekly fields (see example Figure 3). Figure 7d (left, bottom) shows the mean SST from AMSR-E for the year 2005. Finer scales are resolved in this mean field, with strongly meandering paths of the ACC fronts, and sharper gradients across the fronts. In Figure 7c, the mean SST map is computed from the 52 weekly fields of 2005 of Coriolis fields, advected by altimetry over a 14 day period, where the advected velocities are derived from the timevarying absolute dynamic topography. The signatures of the ACC fronts compare well with those in the mean field for AMSR-E SST. For comparison, Figure 7b is similar to Figure 7c, only the advections are performed using time-mean velocities derived from the mean dynamic topography (Rio et al., 2009). Clearly some of the mean SST structures in the AMSR-E data and the fields advected with altimetry (Figures $\mathrm{c}$ and d) are dominated by the permanent mean circulation shown in Figure $7 \mathrm{~b}$, including the double northward meanders at $50^{\circ} \mathrm{S}$ around 145 $150^{\circ} \mathrm{E}$. However, certain structures also have a clear interannual signature which are particular to 2005 , including the strengthening of the jets upstream around $135^{\circ} \mathrm{S}$, and the strong southward meander around $54^{\circ} \mathrm{S}, 150^{\circ} \mathrm{E}$.

We can validate the improvement to the mean fields using the independent satellite SST data, and indeed the similarity between panels $\mathrm{c}$ and $\mathrm{d}$ is quite striking. However, the real benefit for this technique is to improve other tracer fields that cannot be observed from space with sufficient 2D resolution. The right panels in Figure 7 show the equivalent SSS fields. Once again, the lateral advection from altimetry is adding much sharper frontal structures to the larger scale SSS field.

Interannual variations in the frontal positions are evident in the annual mean SST and SSS advected fields, shown for the 5 year period 2002-2007, in Figure 8, and again compared to the AMSR-E SST. The yearly averaged fields have the advantage of reducing part of the advection bias, which has a strong seasonal signal linked to the missing air-sea flux cycle. Finally, the method gives us the potential to calculate these fields over a much longer time period, as long as the initial tracer fields are fairly representative.

\section{Lagrangian advection at the subsurface}

In the above studies we have used Lagrangian advection with altimeter velocities to reconstruct mesoscale structures in various ocean surface tracer fields. While knowledge of mesoscale and submesoscale activity at the ocean surface is important because of its impacts 
on ocean physics and biology, so is the vertical structure of these small scale features in the subsurface, particularly within the mixed layer. The encouraging results obtained at the ocean surface have led us to consider applying the same technique at the subsurface.

The subsurface tracer fields used in this study are the large scale gridded weekly Coriolis fields based on Argo profiles, which are provided at various depths. We use altimeter velocities to advect particles at the subsurface, considering them to be representative of the dynamics within the upper ocean. An example of Lagrangian advection is shown in Figure 9, with various fields simulating the temperature at $400 \mathrm{~m}$ depth on 25 October 2003, and the Coriolis temperature for 22 October 2003 at the same depth. The plots show the gradual stirring of the temperature fields and development of filaments and sharp temperature fronts.

In the absence of 2D fine scale subsurface tracer fields from satellite measurements or other data, we compare the advected Coriolis tracer fields to high-resolution repeat in-situ data, and specifically to eXpendable BathyThermograph (XBT) temperature measurements from the SURVOSTRAL campaign. In Figure 10 we show the temperature measured during a campaign whose track is represented in Figure 9 and which reached $\sim 50^{\circ} \mathrm{S}$ on 25 October 2003, the date simulated in Figure 9 b and c.

The fine scale improvements noted at the surface also apply in the upper ocean layers in this Southern Ocean region. In Figure 10 we can see a strengthening of the signature of the $\mathrm{SAF}$ at $\sim 52^{\circ} \mathrm{S}$ in the advected Coriolis fields, as the advection time increases. As observed at the surface, these visual comparisons show that the ACC fronts are best reproduced for an advection time of $\sim 2$ weeks. In this figure for instance, the 17 day advection is more satisfactory than the 10 day advection for positioning the SAF.

Small scale structures also develop with increasing advection time (Figure 10). A number of small temperature anomalies occur between the SAF $\left(52^{\circ} \mathrm{S}\right)$ and the STF $\left(45^{\circ} \mathrm{S}\right)$ which are not reproduced by the XBT measurements, either at the surface or at $400 \mathrm{~m}$ depth. However, other fine scale structures - such as the narrow peak at $54^{\circ} \mathrm{S}-$ are well simulated. Interestingly, the amplitude and position of the temperature gradient across the SAF is correct and well positioned, yet we have advected the temperature structure using the stronger surface currents rather than the $400 \mathrm{~m}$ depth currents. This may reflect the strong vertical coherence of the currents in these equivalent barotropic jets. In contrast, the narrow peak at $54^{\circ} \mathrm{S}$ is well represented at the surface (Figure 14a), but too strong at $400 \mathrm{~m}$ depth (Figure 14b). Despite using the surface geostrophic velocities for advection, the technique shows some skill for a 
statistical reconstruction of the frontal features at depth, with an optimal advection time of $\sim 2$ weeks. We note that the ageostrophic dynamics which are strong in the surface layer, are much weaker of absent at $400 \mathrm{~m}$ depth, and the geostrophic convergence produces good sharpening of the mean fronts. In future work, we are interested in advecting the subsurface tracer fields with more realistic subsurface currents derived from satellite and in-situ data (e.g., Mulet et al., 2012).

\section{Discussion}

In this regional study we find that Lagrangian surface advection with altimetric geostrophic velocities can improve the representation of ACC fronts and small scale filaments in large scale tracer fields, although with a number of limitations. The passive horizontal stirring leads to increased production of small scale features as we extend the advection time. Comparisons with various high-resolution in-situ or satellite data show that the strong ACC fronts are best reproduced in a statistical sense using an advection time of $\sim 2$ weeks.

We are using a very simple technique, and it is quite remarkable that a simple lateral stirring of large-scale tracer fields with altimetric geostrophic currents can act to sharpen the main circumpolar fronts, and introduce the observed submesoscale structures. This is particularly striking since the individual maps of gridded altimetric data can only resolve horizontal scales of around $150 \mathrm{~km}$. It's the temporal evolution of these currents, driving the geostrophic convergence, which is creating the sharpening of the fronts and realigning their positions. We find that a 15 day advection allows for the best representation of the smaller scales when using Coriolis fields as initial conditions. After advections, the main ACC fronts and finer filaments display generally good gradients and positioning, especially when averaged in a statistical sense.

Our study has also highlighted various factors which contribute to the errors in the instantaneous characteristics of reconstructed fronts. An important source for those errors is the absence of frontogenesis in our method. As flow convergence at a front strengthens the tracer gradients, an ageostrophic circulation appears - which is ignored with this technique that accelerates the frontal generation (Hoskins and Bretherton, 1972). The ageostrophic circulation associated with frontogenesis will also develop faster at a dynamical front if the large scale gradient is strong. Our technique does not include this ageostrophic adjustment, so potentially our optimal advection time based on geostrophic convergence may be longer than 
is really necessary. In addition, our analysis is made over a wide region, which means that all frontal structures may not have identical frontogenesis timescales. Indeed, the geostrophic convergence associated with our technique is only providing one aspect of the frontal dynamics. Another problem concerns the lack of dissipation mechanisms with our technique. Our study shows that long advection times also lead to excessive frontal density and crossfrontal gradients, setting a limit to the advection time that can be used.

Other factors also contribute to errors in the fine scale reconstructions. Here we simulate the passive evolution of a tracer field considering only horizontal geostrophic dynamics resolved with altimetry. In reality, tracers such as SST or SSS will also evolve with air-sea exchanges, vertical movements, diffusion, Ekman transport, or frontogenesis to cite a few factors ignored. Their absence introduces errors in the characteristics of fronts simulated, which we refer to as the advection bias.

In addition to dynamical processes that are left out, the altimetric velocity fields themselves are sources for various errors. Firstly, the technique does not simulate advection by the full surface geostrophic circulation, since only the larger part of the mesoscale circulation is resolved in the altimetric fields. Also, the altimetric velocities do not measure the ageostrophic surface mixed layer currents, but respond to the deeper-reaching geostrophic circulation. Indeed, latitudinal offsets between surface and subsurface positions of ACC fronts have been observed, with northward drifts of surface fronts by the wind-driven Ekman transport (Chaigneau and Morrow, 2002). During summer, there is often a better persistence of the SSS frontal signature than for SST (Morrow et al., 2008), which is also noted in our advected vs. in-situ tracer comparisons in Figure $4-$ particularly for the STF at $\sim 46^{\circ} \mathrm{S}$. Thus the altimetric advection may not be sufficient when the ageostrophic surface mixed layer processes are energetic - this is particularly true in summer, when most of our in-situ observations are available. Despite all of these possible error sources, the observed improvement indicates that these large, deep reaching mesoscale eddies and mean currents have a controlling action in stirring these finer scale fronts and filaments in this part of the Southern Ocean.

It would be tempting to use this method to construct global time-series of tracer maps with finer scales represented. However, our analysis of the errors introduced during advection shows that these errors can be large, and would need to be reduced before producing a time series of finer-scale tracer maps. In particular, the advection bias dominates during periods of 
strong changes in the surface tracer values, and the strong air-sea fluxes are likely the dominant missing factor during those periods. Hence, the overall bias could be significantly reduced by introducing, as a first step, large scale corrections for air-sea fluxes along particle trajectories. Further improvements, either in the general bias or in the positioning and intensity of fronts and filaments, could also be expected by including corrections for Ekman velocities, horizontal diffusion, or vertical mixing during the advection. Also, we find that the biases are smaller in the higher EKE regions, where the geostrophic convergence is stronger. The Southern Ocean is a region of high EKE, with deep-reaching fronts having a strong surface velocity signatures, and the technique may not be as beneficial in less energetic regions.

Apart from the advection technique and its limitations, another factor influencing the overall errors in the advected tracer fields is the quality of the initial tracer fields used. Our study has shown that the large scale Coriolis fields display regional biases. In the southernmost latitudes of the Southern Ocean, very little data is available over a large domain. Hence little to no variability is present in the large scale Coriolis tracer fields, and biases can appear over large domains. As these biases are not associated with the unresolved transient mesoscale features, the horizontal stirring cannot improve this bias (see Figure 4b, south of $\left.54^{\circ} \mathrm{S}\right)$. On the other hand, regions where the Coriolis tracer fields are constructed with more data display smaller large scale bias. The Lagrangian advection appears to work better in such regions.

In this discussion, we have tried to provide a comprehensive coverage of the potential errors in the lateral advection technique. We note however, that much of the validation and intercomparisons are tested against the fine-resolution satellite SST fields, which are the only independent, 2D high-resolution tracer fields available. Yet SST is the most difficult tracer to reconstruct, since it is strongly coupled with the air-sea fluxes, responding quickly to air-sea anomalies and in turn damping the anomalies. The main aim of this technique is to improve the fine-scale structure of other large-scale tracer fields, such as salinity, carbon and nutrients, and these fields may be less sensitive to the air-sea flux forcing over a 15-day period.

We have also applied this technique at the subsurface, advecting large scale Coriolis fields with the same altimetric geostrophic velocity fields. Comparisons with XBT data from SURVOSTRAL cruises show improvements in the SAF gradients and positions, similar to those obtained at the surface. This is explained by the fact that the most energetic frontal 
features in the domain studied - the ACC fronts - are deep-reaching currents, so that the altimeter surface velocities are also representative of the subsurface currents. The advection bias is also smaller in the sub-surface fields since there is no air-sea flux error and less frontogenesis error during the advection time. However, smaller-scale temperature anomalies appeared too strong outside the strongest jets. This subsurface technique would need to be tested in other oceanic regions, corrections to the velocity fields could be required at depth, and a diffusion temer may also be necessary.

Finally, previous studies by Despres et al (2011) used a climatological SSS field as their initial large-scale tracer field for their study in the North Atlantic Ocean, instead of the weekly evolving large scale tracer field used in this study. They obtained good results using this "static" large-scale initial field, stirred weekly with the realistic altimetric currents. Both of these studies indicate that Lagrangian advection could be used to improve the mesoscale to submesoscale representation of any climatologic tracer patterns, as long as horizontal advection dominates the tracer evolution over a short time period, eg 2 weeks. So potentially, the method could be used to introduce sharper frontal structures in global climatological maps of carbon, DMS, nitrate, phosphate, or silicate. Our study also highlights the potential for calculating annual climatologies of SST and SSS, with realistic frontal structures resolved. Indeed, the joint SSS and SST analyses with SURVOSTRAL thermosalinograph measurements show promising results for SSS, especially in the region of the strongest ACC fronts. So the extension to other tracer fields provides an interesting perspective.

\section{Conclusion}

The Lagrangian trajectory calculation technique used in this study shows promising results when applied to the improvement of the horizontal resolution of large scale upper ocean tracer fields. It shows good results in the Southern Ocean, confirming the important role of lateral advection in setting the salinity frontal structure, as found in the North Atlantic Ocean by Despres et al (2011). The technique could also be applied to any tracer that is primarily governed by lateral advection. We find that when starting with large scale tracer fields such as those from Coriolis, a $\sim 15$ day advection time is best able to reproduce the mesoscale and submesoscale features in their statistical positioning and associated gradients. We note that our technique will only provide the geostrophic convergence at the front. 
Missing ageostrophic frontal dynamics would probably reduce this advection time, and may also introduce compensated temperature and salinity gradients at small frontal scales.

Although the technique should only be used for a statistical representation of fronts and filaments, rather than providing a time series of maps, this technique could offer important tracer information. The current need is to improve the resolution of salinity fields to bridge the gap with the better observations of temperature in the upper ocean. This study has shown the promising results for estimated finer-scale salinity, starting from large-scale gridded in-situ salinity fields. In the future, this technique could also be applied to the largescale gridded satellite SST fields from SMOS and Aquarius data, starting with tropical or subtropical regions where the data should have better precision.

One important and largely unknown quantity is the vertical velocity associated with fronts. Vertical velocities are strongly related to density gradients, and hence the tracer gradients across fronts. The statistical reconstruction of SSS and SST, and thus obtaining frontal density, coupled with some knowledge of the vertical velocity profiles at fronts from in-situ observations, could give us valuable insight into vertical dynamics.

While this technique improves the general representation of fine scales, our study shows that errors in front characteristics as well as larger scale errors are introduced during the Lagrangian advection. The advection of the tracer fields is passive, and only takes into account horizontal geostrophic velocities resolved by altimetry. Some elements have been discussed to try to reduce the errors in the advected fields, and in future studies could address the neglected thermodynamical factors, such as air-sea fluxes which are significant in the ocean domain studied, Ekman velocities, horizontal and vertical diffusion and mixing.

An interesting application of the technique is the improvement of the fine scale resolution of climatological tracer fields. The non-mesoscale-resolving fields that were advected for 2 weeks and spatially averaged over a year displayed several fine scale features such as the ACC fronts. Improved surface tracer fields could be useful to validate or initialize model simulations. This technique could provide improved climatologies of any physical or biogeochemical quantity whose evolution is predominantly governed by lateral advection.

\section{Acknowledgments}


This work was financed as a MyOcean research and development study. We gratefully acknowledge the help from Francesco D'Ovidio for the advection code and early assistance with the project. We are also indebted to Elodie Kestenare for her assistance with the SURVOSTRAL TSG data and matlab support, and to Renaud Dussurget of the CTOH/LEGOS for assistance with the altimeter data and code. Two anonymous reviewers also provided helpful and constructive comments.

\section{References}

Abraham, E. R. (1998). "The generation of plankton patchiness by turbulent stirring." Nature 391(6667): 577-580.

Abraham, E. R., C. S. Law, et al. (2000). "Importance of stirring in the development of an iron-fertilized phytoplankton bloom." Nature 407(6805): 727-730.

Belkin, I. M. and A. L. Gordon (1996). "Southern Ocean fronts from the Greenwich meridian to Tasmania." Journal of Geophysical Research-Oceans 101(C2): 3675-3696.

Chaigneau, A. and R. Morrow (2002). "Surface temperature and salinity variations between Tasmania and Antarctica, 1993-1999." Journal of Geophysical Research-Oceans 107(C12).

Cresswell, G. R., (2000): Currents of the continental shelf and upper slope of Tasmania. Pap. Proc. Roy. Soc. Tasmania, 133, 21-30.

d'Ovidio, F., V. Fernandez, et al. (2004). "Mixing structures in the Mediterranean Sea from finite-size Lyapunov exponents." Geophysical Research Letters 31(17): 4.

d'Ovidio, F., J. Isern-Fontanet, et al. (2009). "Comparison between Eulerian diagnostics and finite-size Lyapunov exponents computed from altimetry in the Algerian basin." Deep-Sea Research Part I-Oceanographic Research Papers 56(1): 15-31.

Dencausse, G., M. Arhan, et al. (2011). "Is there a continuous Subtropical Front south of Africa?" Journal of Geophysical Research-Oceans 116.

Despres, A., G. Reverdin, et al. (2011). "Mechanisms and spatial variability of meso scale frontogenesis in the northwestern subpolar gyre." Ocean Modelling 39(1-2): 97-113. 
Dibarboure, G., M. I. Pujol, et al. (2011). "Jason-2 in DUACS: Updated System Description, First Tandem Results and Impact on Processing and Products." Marine Geodesy 34(3-4): 214241.

Dussurget, R., F. Birol, et al. (2011). "Fine Resolution Altimetry Data for a Regional Application in the Bay of Biscay." Marine Geodesy 34(3-4): 447-476.

Ferrari, R., J. C. McWilliams, V. Canuto, and D. Dubovikov, 2008: Parameterization of eddy fluxes at the ocean boundaries, J. Climate, Vol. 21, 2770-2789.

Fox-Kemper, B., R. Ferrari, et al. (2008). "Parameterization of mixed layer eddies. Part I: Theory and diagnosis." Journal of Physical Oceanography 38(6): 1145-1165.

Guinehut, S., P. Y. Le Traon, et al. (2004). "Combining Argo and remote-sensing data to estimate the ocean three-dimensional temperature fields - a first approach based on simulated observations." Journal of Marine Systems 46(1-4): 85-98.

Hoskins, B. J., F. P. Bretherton, 1972: Atmospheric Frontogenesis Models: Mathematical Formulation and Solution. J. Atmos. Sci., 29, 11-37.

Hughes, C. W. and E. R. Ash (2001). "Eddy forcing of the mean flow in the Southern Ocean." Journal of Geophysical Research-Oceans 106(C2): 2713-2722.

Le Sommer, J., F. d'Ovidio, et al. (2011). "Parameterization of subgrid stirring in eddy resolving ocean models. Part 1: Theory and diagnostics." Ocean Modelling 39(1-2): 154-169.

Le Traon, P. Y., G. Dibarboure, et al. (2001). "Use of a high-resolution model to analyze the mapping capabilities of multiple-altimeter missions." Journal of Atmospheric and Oceanic Technology 18(7): 1277-1288.

Lehahn, Y., F. d'Ovidio, et al. (2007). "Stirring of the northeast Atlantic spring bloom: A Lagrangian analysis based on multisatellite data." Journal of Geophysical Research-Oceans 112(C8).

Levy, M., M. Gavart, et al. (2005). "A four-dimensional mesoscale map of the spring bloom in the northeast Atlantic (POMME experiment): Results of a prognostic model." Journal of Geophysical Research-Oceans 110(C7). 
Levy, M., P. Klein, et al. (2001). "Impact of sub-mesoscale physics on production and subduction of phytoplankton in an oligotrophic regime." Journal of Marine Research 59(4): $535-565$.

Morrow, R., A. Brut, et al. (2003). "Seasonal and interannual variations of the upper ocean energetics between Tasmania and Antarctica." Deep-Sea Res. Part I. 50(3): 339-356.

Morrow, R., Valladeau, G., and Sallee, J. (2008). Observed subsurface signature of Southern Ocean decadal sea level rise. Prog. Oceanogr. Vol 77/4 pp 351-366

Mulet, S., et al., A new estimate of the global 3D geostrophic ocean circulation based on satellite data and in-situ measurements. Deep-Sea Res. II (2012), http://dx.doi.org/10.1016/j.dsr2.2012.04.012

Orsi, A. H., T. Whitworth, et al. (1995). "On the meridional extent and fronts of the Antarctic Circumpolar Current." Deep-Sea Res. Part I 42(5): 641-673.

Paci, A., G. Caniaux, et al. (2005). "A high-resolution simulation of the ocean during the POMME experiment: Simulation results and comparison with observations." Journal of Geophysical Research-Oceans 110(C7).

Rio, M. H., P. Schaeffer, et al. (2009). A new mean dynamic topography computed over the global ocean from GRACE data, altimetry and in-situ measurements. Poster communication at OceanObs09 symposium, 21-25 September 2009, Venice, Italy.

Sallee, J.-B., N. Wienders, et al. (2006). "Formation of subantarctic mode water in the southeastern Indian Ocean." Ocean Dynamics 56(5-6): 525-542.

Sallee, J. B., K. Speer, et al. (2008). "An estimate of Lagrangian eddy statistics and diffusion in the mixed layer of the Southern Ocean." Journal of Marine Research 66(4): 441-463.

Sokolov, S. and S. R. Rintoul (2007). "Multiple jets of the Antarctic circumpolar current South of Australia." Journal of Physical Oceanography 37(5): 1394-1412.

Thomas, L. and R. Ferrari, 2008: Friction, frontogenesis, frontal instabilities and the stratification of the ocean surface mixed layer, J. Phys. Oceanogr., Vol. 38, 2501-2518. 
Turiel, A., J. Isern-Fontanet, et al. (2005). "Multifractal method for the instantaneous evaluation of the stream function in geophysical flows." Physical Review Letters 95(10).

Waugh, D and E.R. Abraham (2008). Stirring in the global surface ocean. Geophys. Res. Lett., 35, doi:10.1029/2008GL035526 


\section{Appendix : Refined analysis of optimal advection time}

Our first visual analyses indicate an optimal advection time of $\sim 2$ weeks. We also examined how the bias between the advected tracer values and the in-situ data varies in relation to the advection time - over the entire domain or within the 3 subdomains shown in Figure 5. However, no clear minimum bias appears for a specific advection time, over any domain. This is partly explained by the fact that the underway in-situ measurements only cover 4-5 months of the year. Since there is a seasonal cycle in the bias, a significant advection bias is introduced by the advection technique, because the missing physics (eg airsea fluxes) depend on the time of year. Thus a better way to search for an optimal advection time in a quantitative manner is to make comparisons with data over a full year in order to average out the seasonal component of the bias. To do so, we use a time series of composite high resolution SST infrared data (AVHRR).

For this analysis, we consider the year 2005 and the domain $132-152^{\circ} \mathrm{E}$ and $43-58^{\circ} \mathrm{S}$. Figure 5 had shown that the SST bias was among the smallest for that year, so the large scale Coriolis fields seem the most accurate. We ran 4 sets of advections of Coriolis SST fields with advection times of 7, 14, 21 and 28 days over the domain. For each set, 52 weekly advections are calculated. Each one is obtained by advecting the Coriolis SST fields over the given advection time ( 1 to 4 weeks) up until the simulated date. Then, for each of the 52 dates of a set, averages of advected Coriolis SST and of AVHRR SST are computed for each $1^{\circ} \times 1^{\circ}$ cell, and their difference gives the bias. To obtain the average optimal advection time in each cell of the domain studied, we first calculate the yearly mean bias for each cell and for each advection time. We then determine, for each cell, which advection time minimizes the yearly mean bias.

The histogram of optimal advection time Figure A.1a shows a local maximum at 14 days, confirming our previous qualitative observations. However, this result is not entirely conclusive, as advection times of 0 and 28 days are the most frequent over the domain. Indeed, the 2D map of optimal advection time Figure A.1b shows organized patterns, with regions of 0 and 28 days regrouped into patches.

Previous studies of similar surface Lagrangian advections have shown that most submesoscale filaments and fronts are simulated in the vicinity of mesoscale eddies resolved in the velocity fields used for advection (d'Ovidio et al., 2004; Waugh and Abraham, 2008). 
To explore the influence of mesoscale activity on the choice of an optimal advection time, we compute the annual mean eddy kinetic energy (EKE) for 2005 (Figure A.2a) and plot the distribution of EKE versus optimal advection time over all $1^{\circ} \times 1^{\circ}$ cells (Figure A.2b). The EKE map shows a region of higher EKE east of Tasmania, explained by the fluctuating southward penetration of the East Australian Current Extension. Regions of higher EKE are also observed along $\sim 52^{\circ} \mathrm{S}$, and maximum EKE is located in the southeastern part of the domain studied. This high EKE can be explained by intense eddy activity as well as the meandering of the principal ACC fronts around quasi-stationary paths (Morrow et al, 2003, Sokolov and Rintoul, 2007).

The distribution of EKE vs. optimal advection time shows that regions of moderate to high EKE are on average associated with a 14 day optimal advection time. This agrees with previous studies based on Finite Time Lyapunov Exponents (FTLE) and advection from altimetric currents (eg Abraham and Bowen, 2002; Waugh and Abraham, 2008). For short advection times (eg 5 days), the stirring by FTLEs is dominated by the pure local strain rate, for longer advection times (10-20 days), an integrated stirring along the Lagrangian trajectory occurs and the stirring tends towards a homogenised and stable limit.

In our analysis, frequent optimal advection times are also found at the extremes: 0 and 28 days, associated with regions of low EKE. Regions of low eddy activity are generally associated with larger scale surface tracers patterns and weaker tracer gradients, and the weaker advective stirring does not bring any improvement - in terms of any tracer bias found here. This may explain why regions of 0 day optimal advection times (see white contours in Figure A2a) are generally regions of low EKE.

A 28 day optimal advection time is observed over $\sim 25 \%$ of the domain. These cases also have low eddy activity, and we would expect an optimal advection time of zero days in these regions as well. However, depending on the time of year - and thus the sign of the temporal gradient of SST - the advection bias can help reduce a large tracer bias. When that occurs, the longer advection may improve the tracer bias, resulting in an optimal 28 day advection time (the longest advection time considered). Tracer bias depends on the quantity and quality of data available. As these vary in both space and time, so does the tracer bias, resulting in a more complex pattern in the 28 day optimal advection time distribution.

For this optimal advection time calculation, we note that the convergence due to the ageostrophic flow at the front is not included. Given the same convergence flow and including 
ageostrophic adjustment, fronts will develop faster if there is a strong large-scale density contrast, than if the density contrast is weak. This is not occurring with our technique. So in order to generate fronts of the observed magnitude, the Lagrangian advection has to be extended for a longer time period in the presence of strong density gradients. Thus we may be overestimating the optimal advection time in regions of strong density gradients, often with moderate to high EKE.

In conclusion, the optimal advection time of $\sim 14$ days seen in the summer months in comparison to SURVOSTRAL in-situ data appears robust over the yearly averages, for moderate to high eddy energy regions. Since we do not include the ageostrophic circulation, this is probably overestimating the frontogenesis timescale. For low energy regions, the horizontal stirring is not efficient in correcting the background biases. 


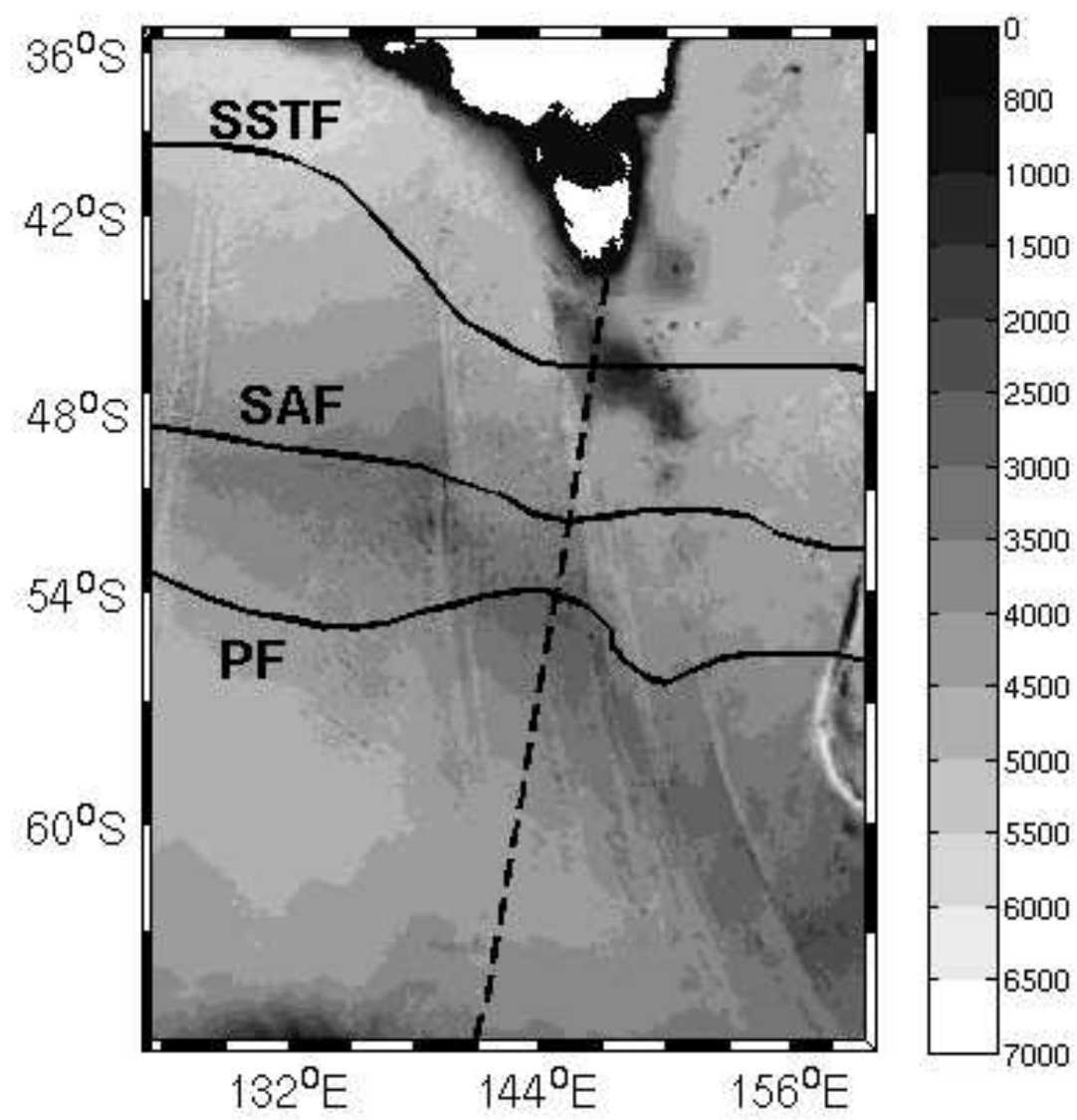

\section{FIGURE 1}

The mean tracks of the South Subtropical Front (SSTF), the Subantarctic Front (SAF) and the Polar Front (PF) from Belkin and Gordon (1996) are represented over a bathymetric map of the study region. The dashed line shows the mean path of the SURVOSTRAL cruise over 2002-2007. 

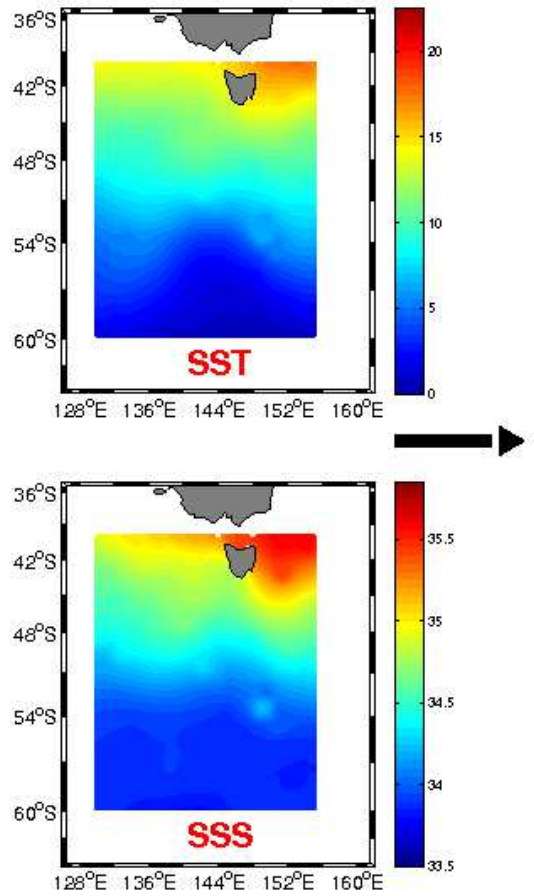
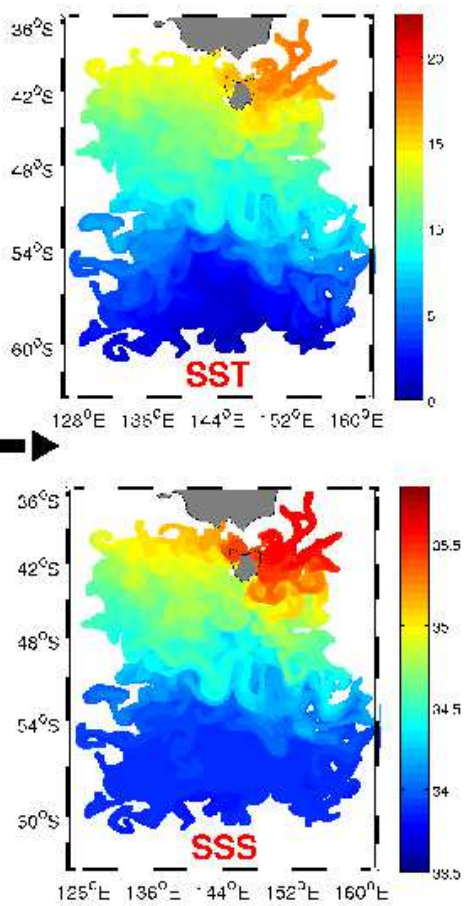

FIGURE 2

Schematic diagram of the Lagrangian advection technique. The central figure shows particles placed on a dense regular grid $\left(0.04^{\circ}\right)$ which are advected horizontally using altimetric geostrophic velocities. The velocity fields along the particle trajectories are calculated every 3 hours from a linear interpolation of the weekly gridded altimetric fields. Large scale tracer fields (e.g.SST and SSS on the left) are interpolated onto each initial particle position. Tracer values are then carried by each particle onto their final position (right panel), to create an 'advected tracer field'. The advection time in this example is 19 days, from 1 January to 20 January 2007. 

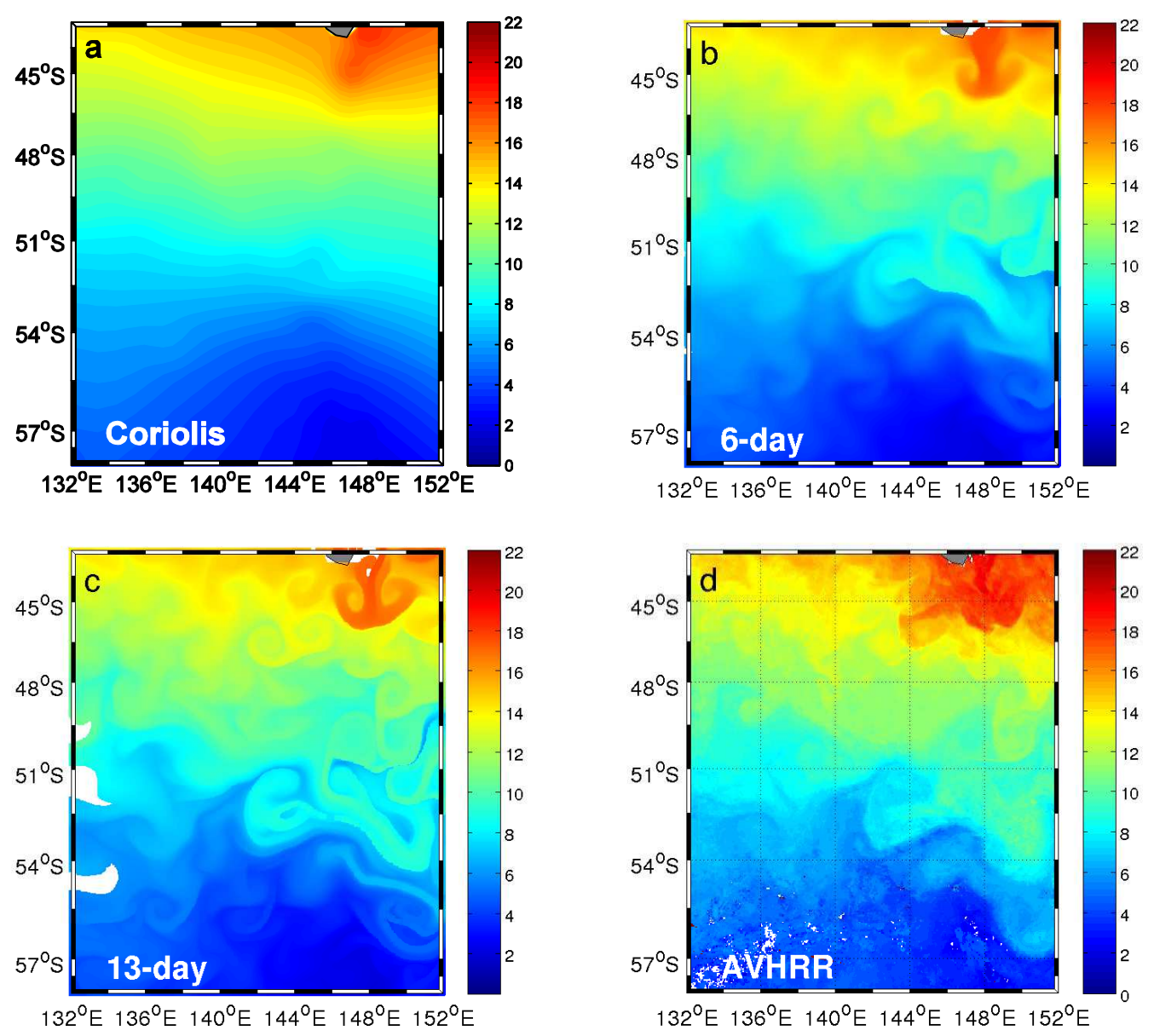

\section{FIGURE 3}

All 4 panels correspond to SST on 20 February 2007. a) Large scale SST from Coriolis objective analysis of in-situ data. b) Final SST after a 6 day advection of the Coriolis field starting from 14 February 2007. c) Final SST after a 13 day advection of the Coriolis field starting from 7 February 2007. d) AVHRR high resolution image. 

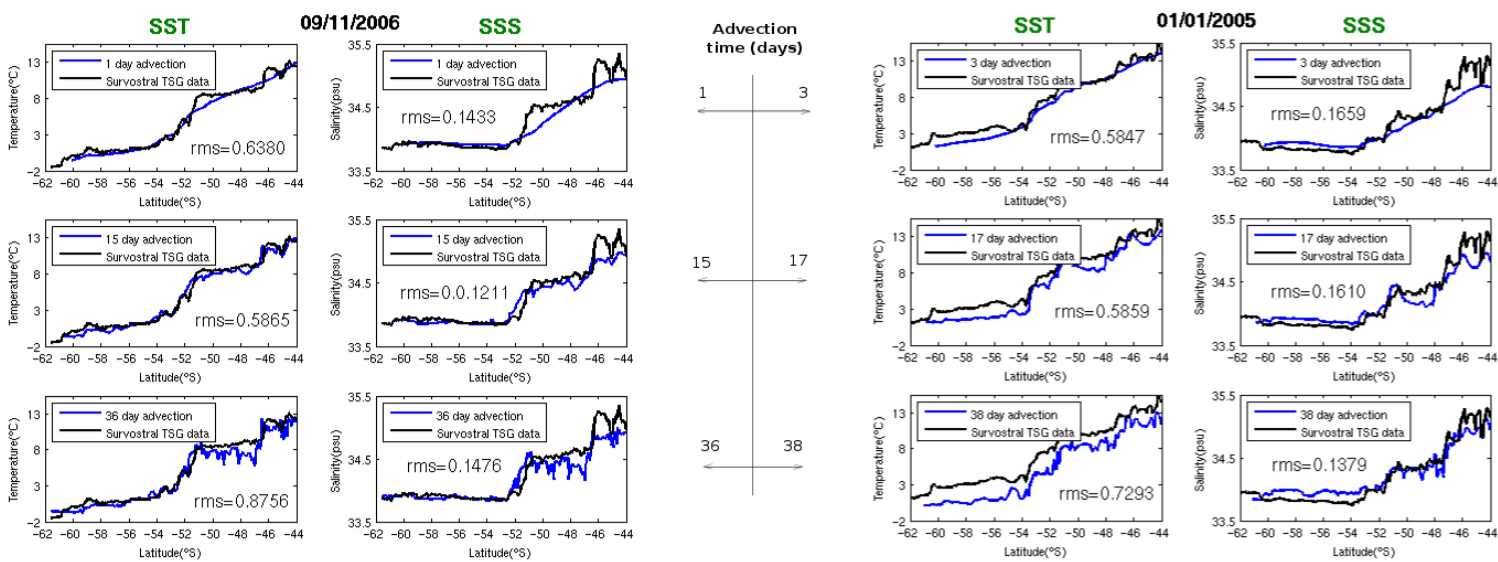

\section{FIGURE 4}

Left panels correspond to SURVOSTRAL campaigns centred on 9 November 2006. Right panels for 1 January 2005. For each panel, SSS and SST values are plotted along the ship track. Black lines are for observed thermosalinograph values, while blue lines correspond to the values obtained by advecting the Coriolis fields. For each tracer, three plots are shown. The thermosalinograph values are unchanged, but the advection time for the Coriolis fields increases from top to bottom. The rms of the difference between the two curves in each plot are shown. 
SSS

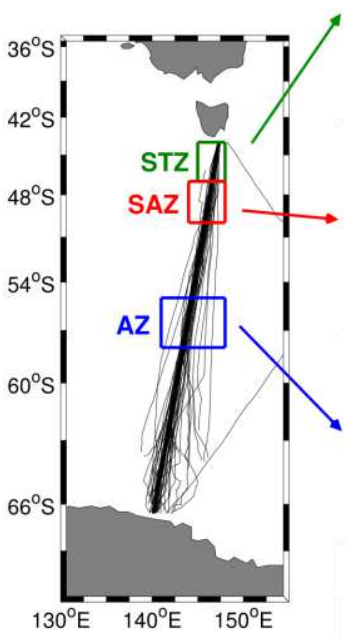

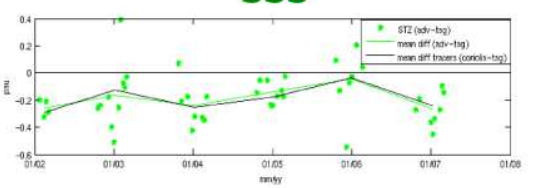
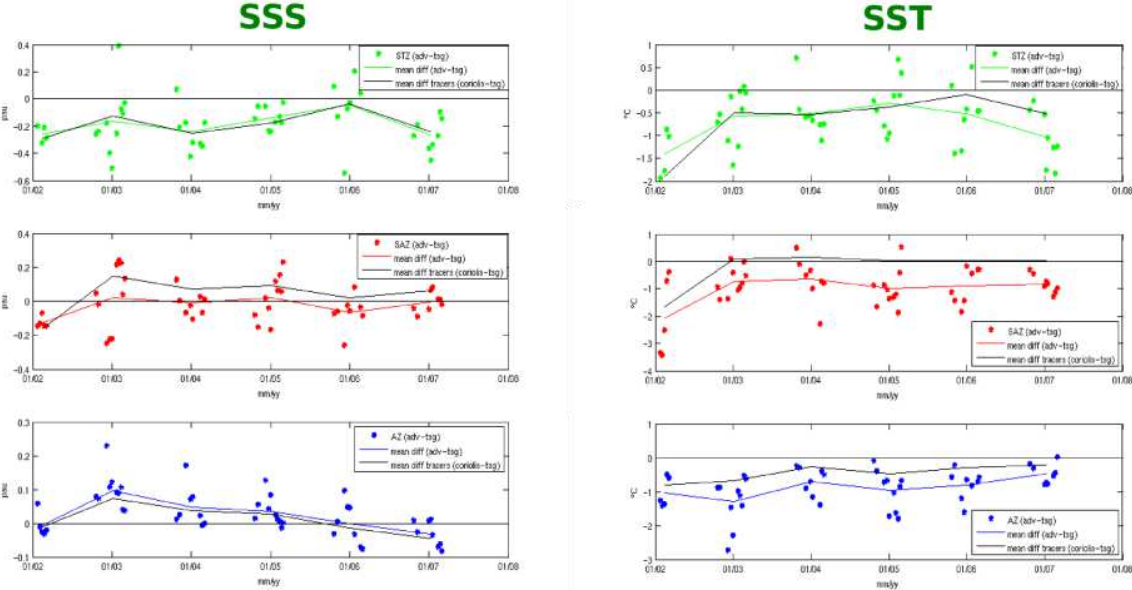

Time evolution in AZ
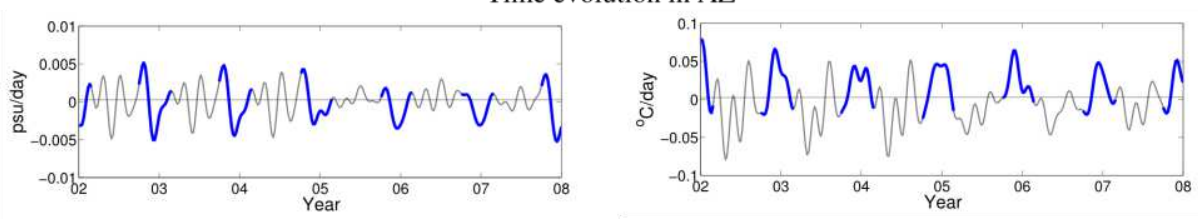

FIGURE 5

Left) SURVOSTRAL ship transects performed over 2002-2007 are plotted, along with the rectangular domains referred to as the Subtropical, Subantarctic and Antarctic Zones (STZ, $\mathrm{SAZ}$ and AZ respectively). The coloured dots of the top 6 panels show the time evolution of the mean SSS (centre) and SST biases (right panels) between the advected Coriolis fields and the thermosalinograph measurements in each domain (the advection time is 14 days $( \pm 3)$ ). In each panel, the coloured line shows the evolution of the mean bias over all transects of the yearly campaigns, while the black line shows the evolution of the estimated tracer bias over the campaign periods. The 2 bottom panel shows the time derivative of the SSS and SST, spatially averaged over the AZ for the Coriolis fields over the 5 year period (60-day low-pass filtered). Each summer SURVOSTRAL period is marked in blue. 

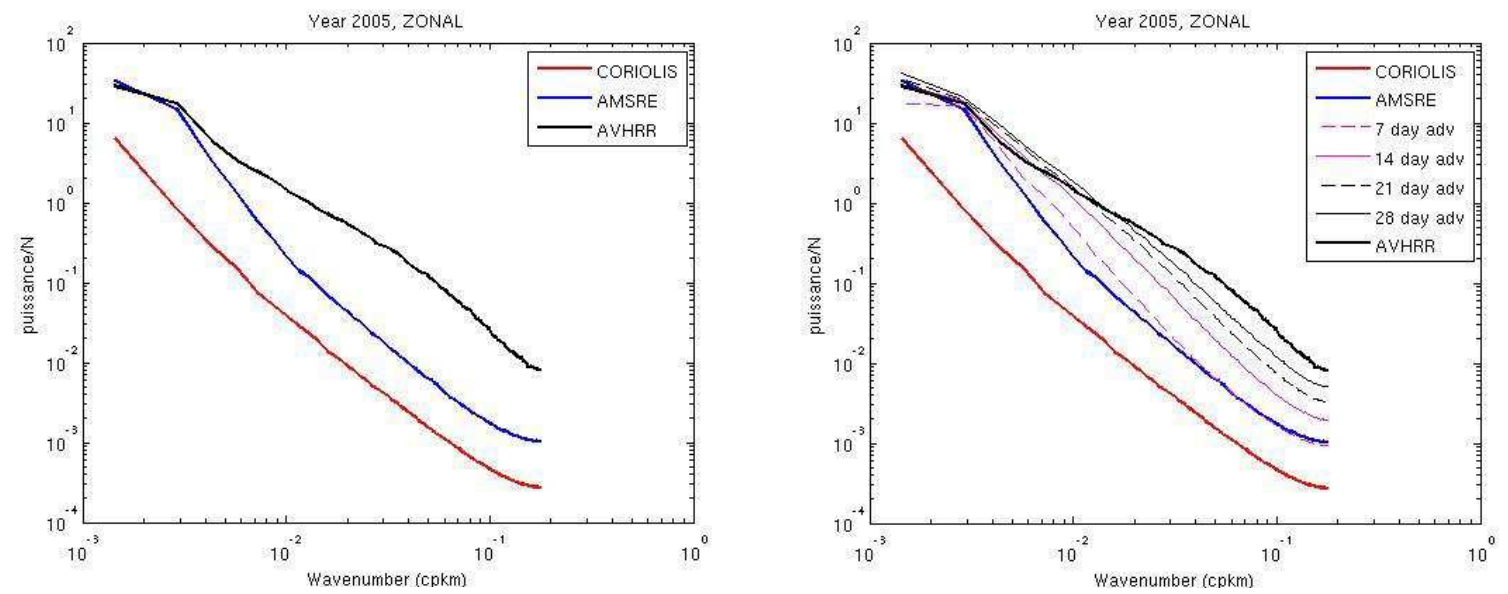

FIGURE 6

Both panels show mean longitudinal power spectra of SST fields for the year 2005, computed over the domain [141-151 ${ }^{\circ} \mathrm{E}$ and $\left.46-56^{\circ} \mathrm{S}\right]$ Left) Spectra show the Coriolis large scale SST field(red), the AMSRE microwave SST field (blue) and the AVHRR high resolution 6-day composite fields (black). Right) The same spectra as in left, plus the advected Coriolis fields, for advection times of 7, 14, 21 and 28 days. 

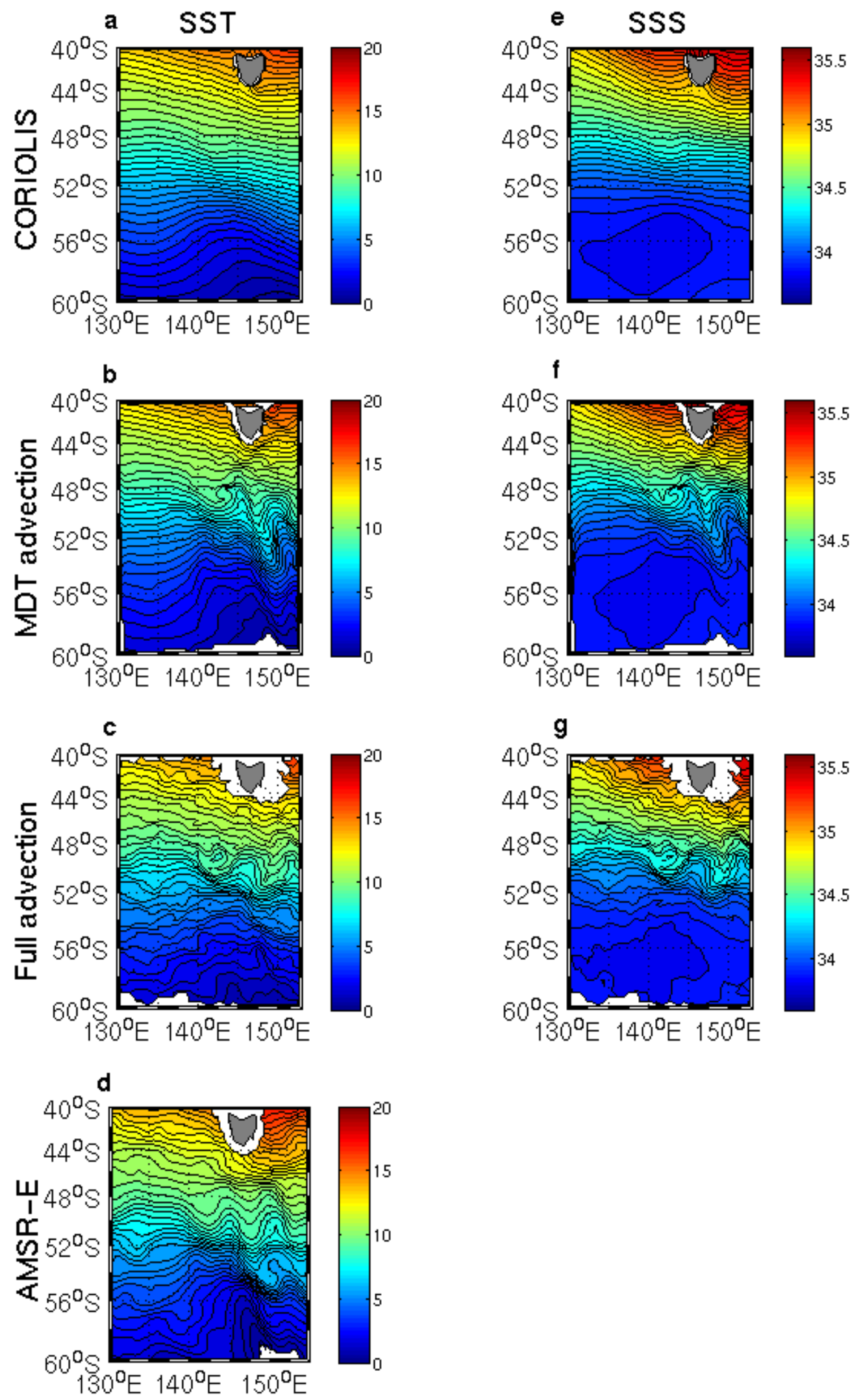

FIGURE 7 
All panels show mean tracer fields for the year 2005, panels a) to d) on the left showing SST and e) to g) on the right showing SSS. The mean fields correspond to Coriolis SST and SSS (a \& e), their 2 week Lagrangian advection with mean velocities only "MDT advection" (b \& f)) derived from the mean dynamic topography (Rio et al., 2009), and their advection with altimetric geostrophic velocities derived from the absolute dynamic topography "Full advection" (c) \& g)). b) This panel shows the mean SST from AMSR-E microwave data. 

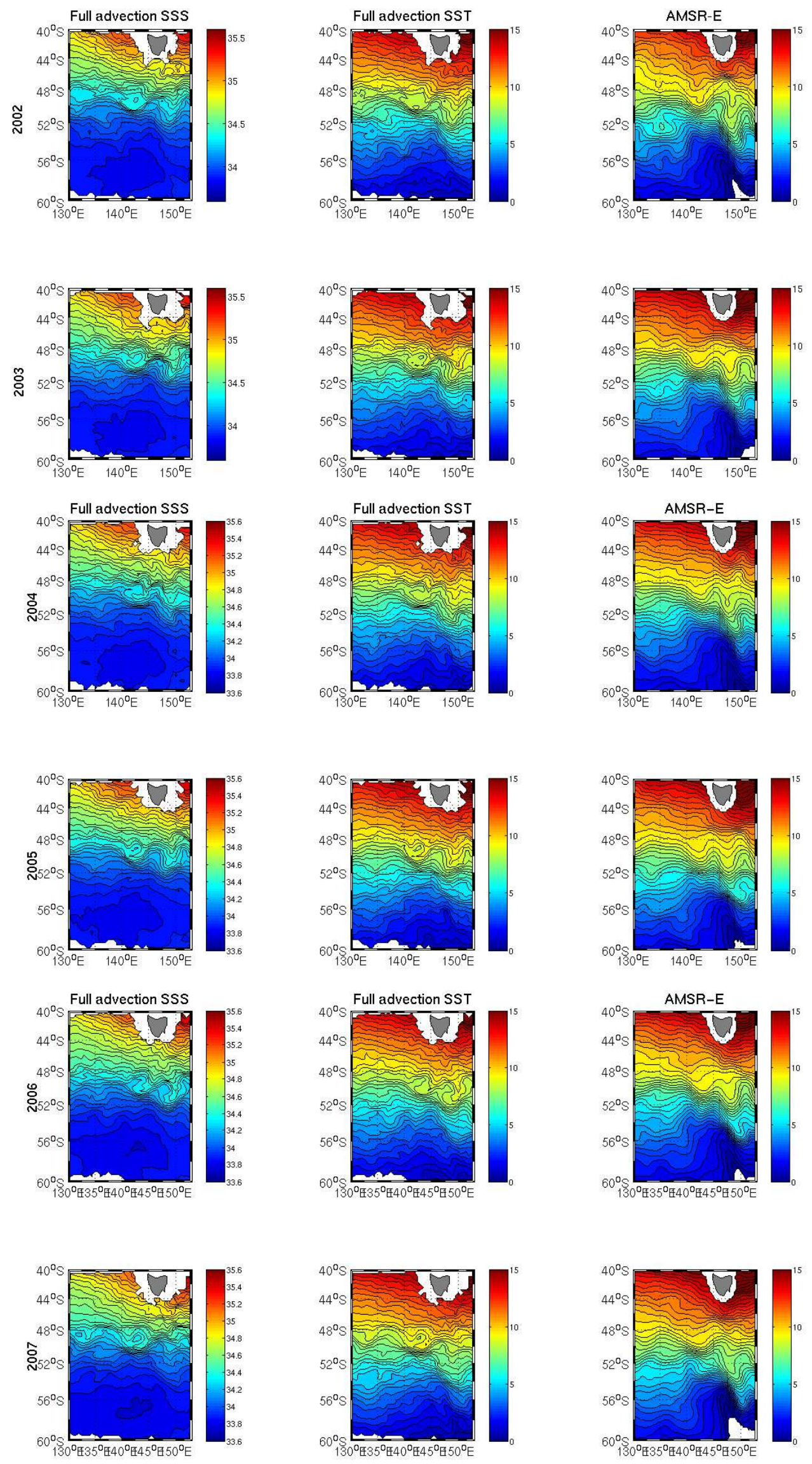


\section{FIGURE 8}

Plots of the climatological fields for years 2002 to 2007 from top to bottom. For each year, the fields shown correspond to the full advection of SSS (left) and SST(centre), and of AMSR-E SST. 

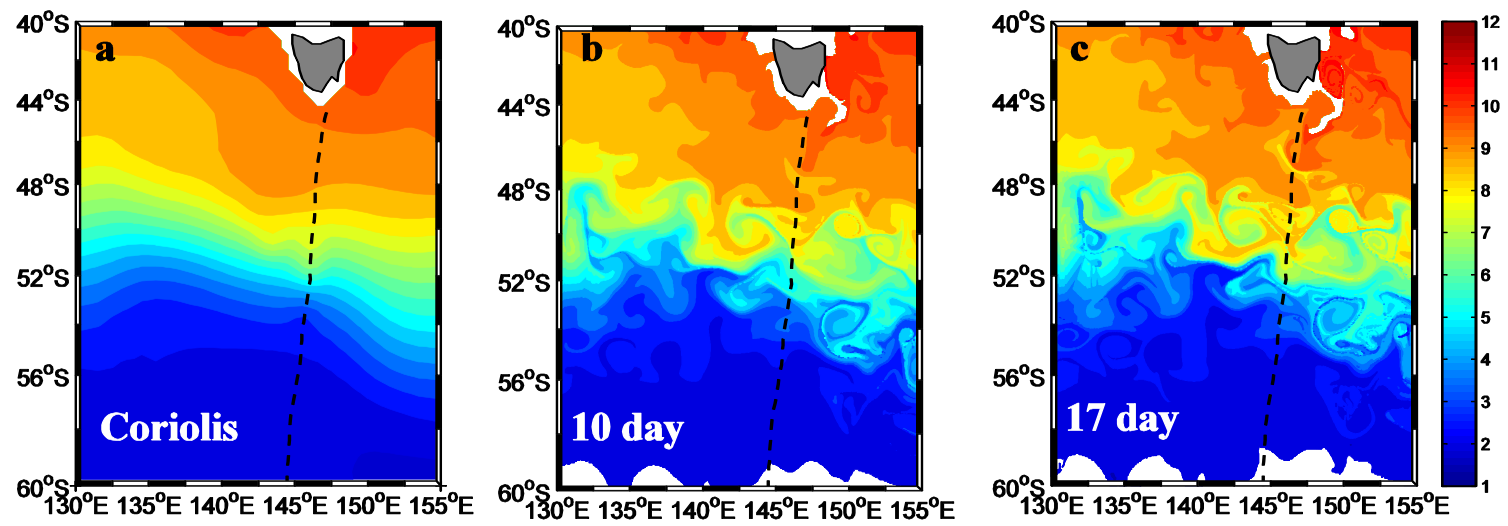

FIGURE 9

Panels a to c show various temperature fields at $400 \mathrm{~m}$ depth. a) is the Coriolis SST field for 22 October 2003, while b) and c) show the simulated fields, for 25 October 2003, obtained with a Lagrangian advection of Coriolis fields over 10 and 17 days respectively. The dotted line shows the path of a SURVOSTRAL transect which reached $\sim 50^{\circ} \mathrm{S}$ on 25 October 2003. 

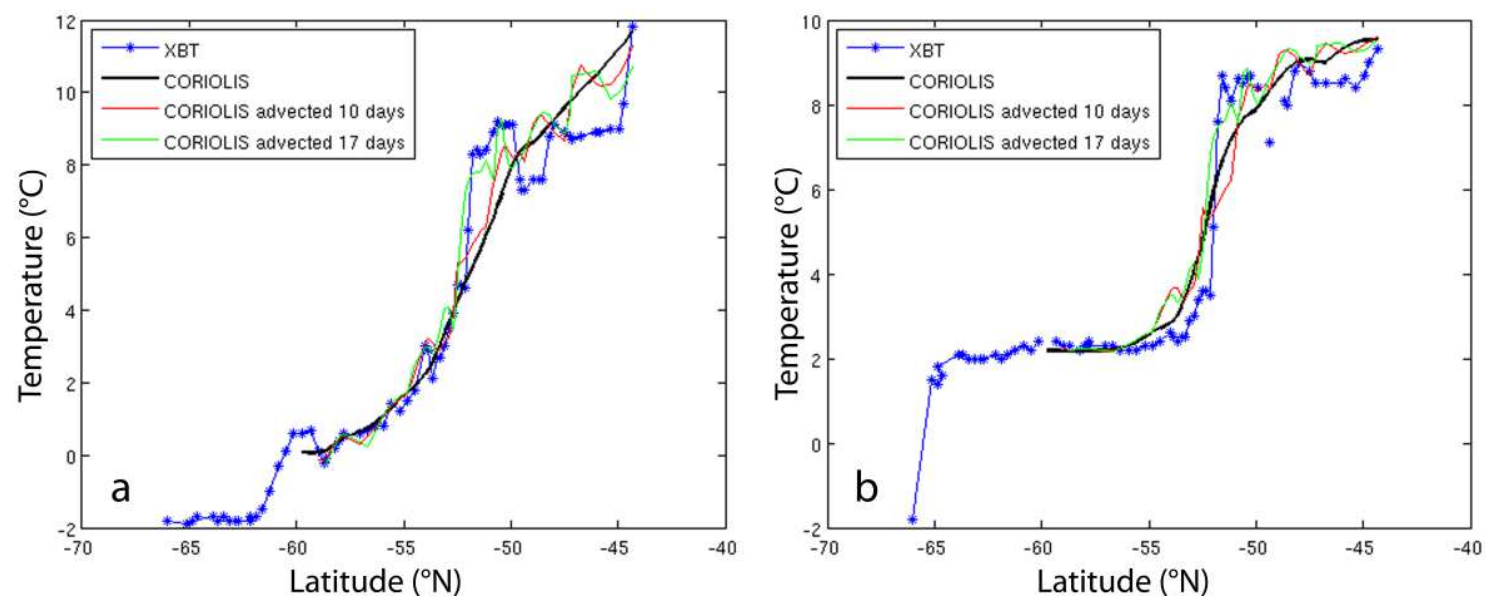

FIGURE 10

The panels show temperature values at a) the surface and b) $400 \mathrm{~m}$ depth along the ship track of a SURVOSTRAL campaign centred on 22 October 2003. Plotted are the observed XBT values (blue line and asterisks), the large-scale Coriolis temperature field for 22 October 2003 (black line) and the 10-day (red) and 17-day (green) advections of Coriolis temperature fields up until 25 October 2003. The Coriolis fields are projected along the XBT cruise track shown in Figure 11 ( $a, b$ and $c$ respectively). 

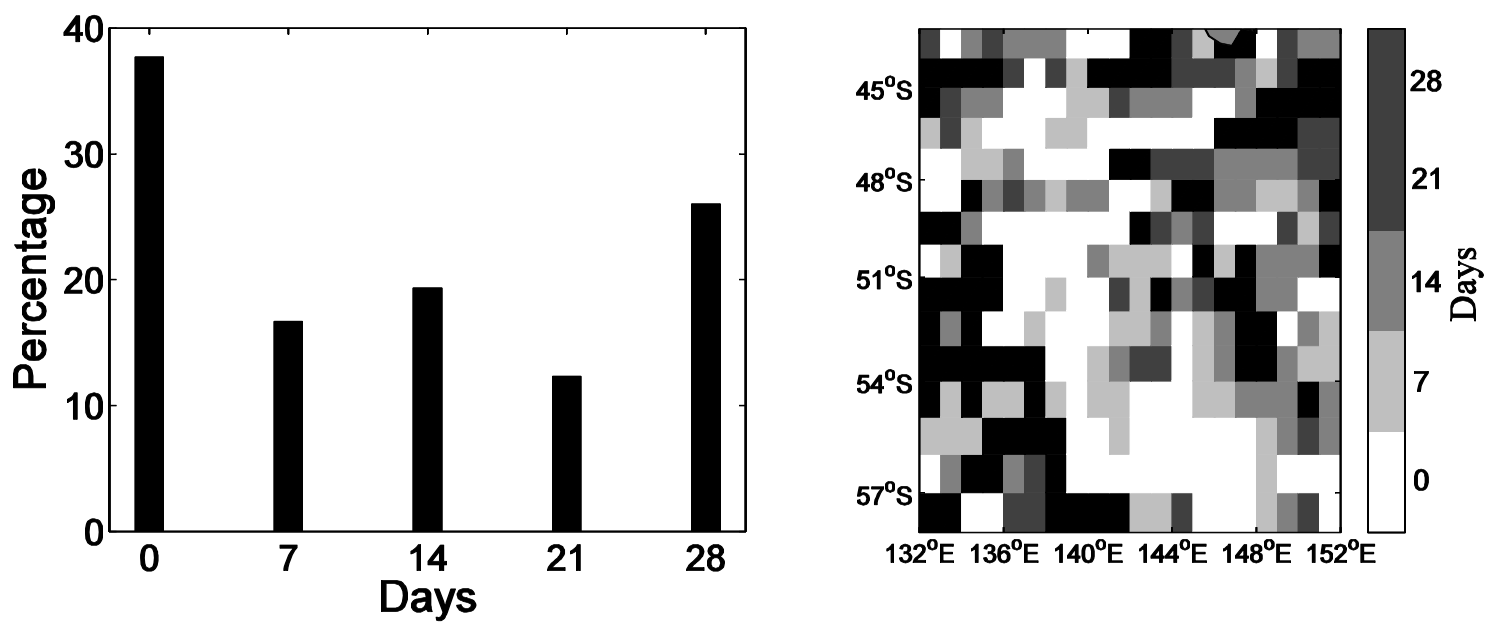

FIGURE A1

Both panels are distributions of the optimal advection time - for $1^{\circ} \times 1^{\circ}$ cells of the domain [132- $152^{\circ} \mathrm{E}$ and $43-58^{\circ} \mathrm{S}$ ] for the year 2005 - considering advection times of $0,7,14,21$ and 28 days (see text for further details). a) Percentage of the total $1^{\circ} \times 1^{\circ}$ in the domain studied for each optimal advection time. b) Geographical distribution of the optimal advection times.
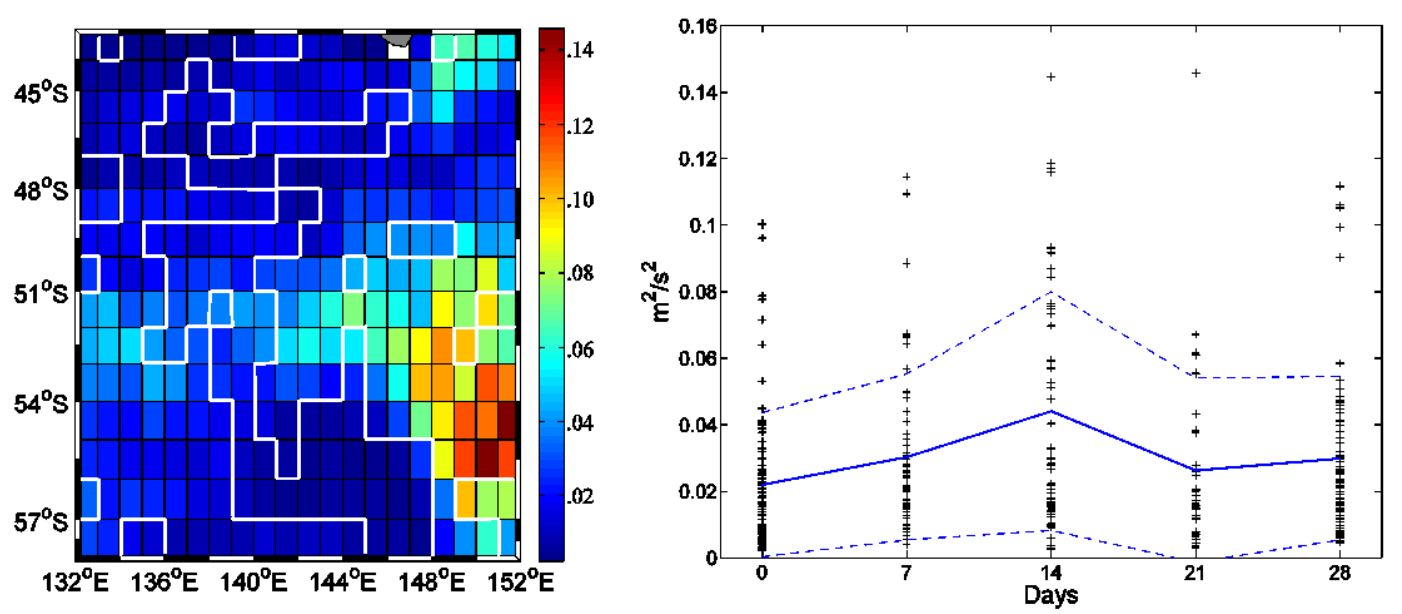

FIGURE A2

a) Mean EKE for the year 2005 computed from the weekly Aviso 1/3 degree fields, and averaged over $1^{\circ} \times 1^{\circ}$ cell. White contours show regions of 0 day optimal advection time (see Figure A1b). b) The distribution of optimal advection time over 2005 for each cell of the same domain versus mean EKE. The blue line shows the mean EKE for each advection time, with the dashed blue lines at \pm one standard deviation. 